\title{
Adjunct Use of Therapeutic Scleral Lens for Exposure Keratopathy after Severe Chemical Burn
}

\author{
Angelica C. Scanzera ${ }^{a}$ b Amjad Ahmad ${ }^{c}$ Ellen Shorter ${ }^{a}$ \\ aDepartment of Ophthalmology and Visual Sciences, University of Illinois at Chicago, \\ Chicago, IL, USA; ${ }^{b}$ Division of Epidemiology and Biostatistics, School of Public Health, \\ University of Illinois at Chicago, Chicago, IL, USA; ' ${ }^{A}$ dvocate Health Care, Chicago, IL, USA
}

\section{Keywords}

Chemical burn $\cdot$ Corneal trauma $\cdot$ Scleral lens

\begin{abstract}
Severe chemical burns can damage the periocular area causing exposure keratopathy and resulting in ocular pain, foreign body sensation, epiphora, photophobia, and vision loss. A custom fit large diameter therapeutic scleral lens can protect the ocular surface by preventing desiccation and providing immediate improvement in comfort. This case describes the management of a patient with extensive chemical burn with complete lid loss and exposure keratopathy who has maintained excellent visual acuity and comfort with therapeutic scleral lens use.
\end{abstract}

\section{Introduction}

Chemical burns from hydrofluoric acid can cause immediate and devastating tissue destruction, and morbidity depends on timeliness of treatment. Despite reconstructive surgery with tissue grafting, lid loss can lead to long-term complications and loss of vision due to exposure keratopathy. Therapeutic scleral lenses can provide protection to the ocular surface, improving visual acuity and quality of life. This case report highlights the use of a therapeutic scleral lens to manage exposure keratitis due to extensive chemical burn and periocular tissue loss. The evaluation of protected health information was in compliance with HIPAA and the Declaration of Helsinki. 


\section{Case Report/Case Presentation}

A 41-year-old white-male long-haul trucker presented for evaluation due to complications after severe chemical burn involving the right side of his face. He had resultant eyelid loss and exposure keratitis affecting the right eye. Twelve years prior, he had a motor vehicle accident with extensive hydrofluoric-acid chemical burn (shown in Fig. 1a). At the time of the accident, he was immediately transferred to a hospital and his ocular surface was thoroughly irrigated with a Morgan lens. Slit lamp examination revealed a large corneal abrasion and scattered superficial punctate keratopathy. He was treated with cycloplegia, erythromycin ointment, and frequent artificial tears. Upon healing of the abrasion, a 12-day steroid treatment with taper was initiated with good results. Two days later, he underwent partial-thickness skin grafting to the upper and lower eyelids (shown in Fig. 1b).

At presentation, his chief complaints were right eye pain with light sensitivity, excessive tearing, and headaches despite use of a therapeutic soft lens, frequent preservative-free artificial tears, UV protection, nightly erythromycin ointment, and overnight moisture chamber use. Best-corrected visual acuity was 20/40 in the right eye. There was marked irregular corneal astigmatism with simulated keratometry readings of 44.95/41.07 with inferior nasal elevation. Anterior segment evaluation of the right eye revealed absent upper and lower lid margins with neither lashes nor puncta, with grade 1 diffuse injection and exposed bulbar conjunctiva. The cornea had grade 2 diffuse punctate epithelial erosions and a 3.5 by $4.3 \mathrm{~mm}$ dellen with $30 \%$ stromal thinning. Bell's reflex was intact (shown in Fig. 2a). A 15-mm diagnostic scleral lens (Essilor Jupiter XO 46D -6.00 $15 \mathrm{~mm}$ ) was fit after a larger 18.2 diameter lens was unsuccessful due to insufficient fornix space and discomfort (shown in Fig. 2b). A spherical overrefraction improved the patient's vision to $20 / 30$. He was trained on safe lens application with nonpreserved saline for daily use with nightly disinfection with hydrogen peroxide-based system. Potential risks including midday lens fogging, inflammation, and infection were discussed.

After 3 months of daily scleral lens wear, best-corrected visual acuity was 20/20 and the corneal punctate epithelial erosions resolved. Despite severe eyelid abnormalities, he reported improved comfort, decreased dryness, fewer headaches, and less light sensitivity.

\section{Discussion}

Chemical burns from hydrofluoric acid cause immediate tissue destruction through 2 mechanisms: corrosive hydrogen ion activity causes immediate tissue destruction, and fluoride ions cause liquefaction necrosis to deep tissues [1]. The morbidity of the chemical
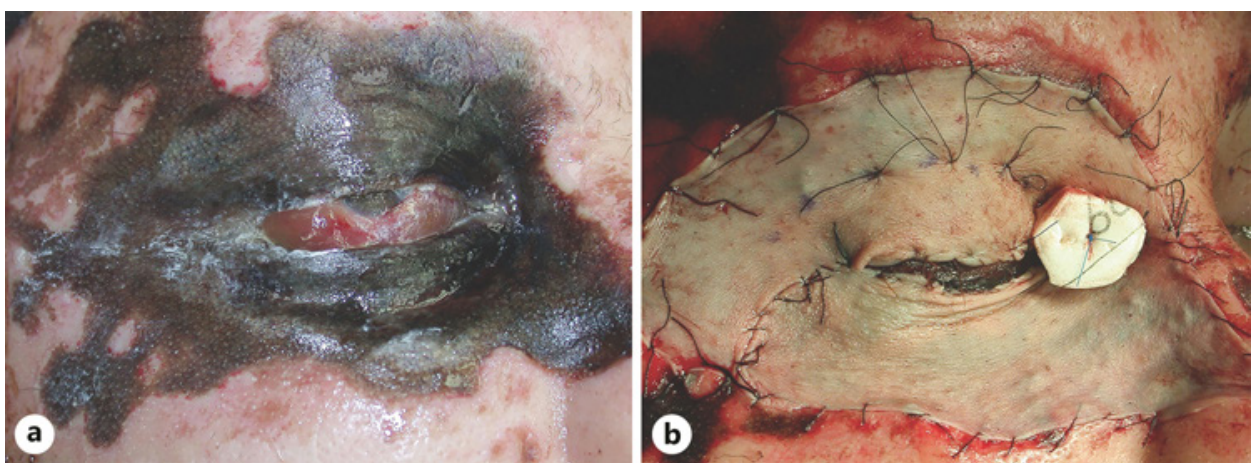

Fig. 1. a Initial chemical burn with extensive necrotic tissue. b Intraoperative partial-thickness skin grafting (3 days after injury).

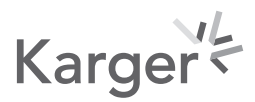



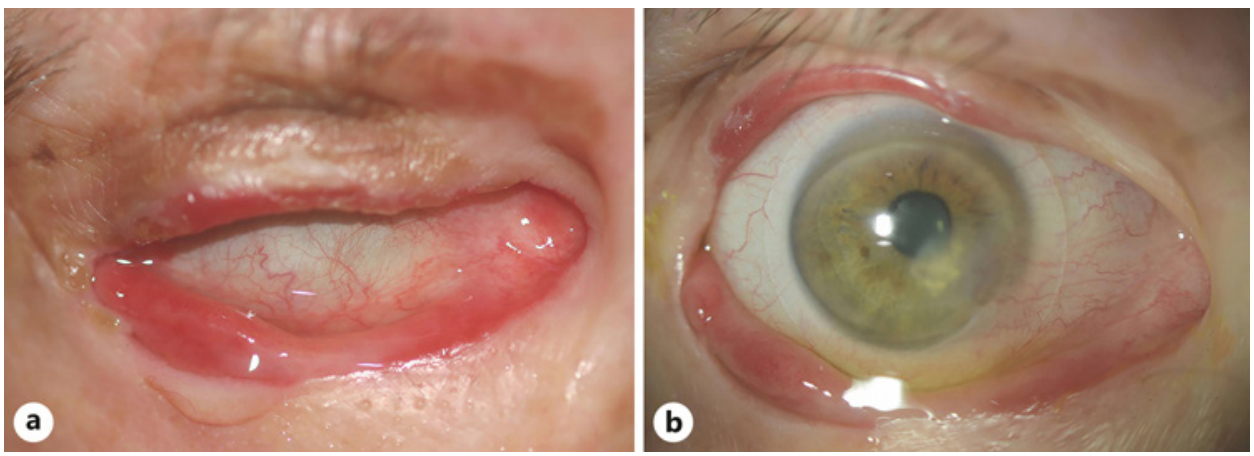

Fig. 2. a Intact Bell's reflex. b Image of therapeutic scleral lens on the right eye.

burn is directly proportional to the concentration, duration of exposure, and timeliness of treatment [1]. Chemical injuries involving the eyes and eyelids can result in severe sequelae including exposure keratopathy. Ocular chemical burns make up about 7.7-18\% of all ocular traumas [2] and comprise almost 16,000 cases in the U.S. each year [3]. Sequelae include changes to the lids, such as lagophthalmos, trichiasis, ectropion, or entropion [4]. In addition, changes to the ocular surface include dry eye, corneal melt, scarring, nonhealing epithelial defects, loss of goblet cells, limbal stem cell deficiency, and recurrent corneal erosions [4]. Treatment of exposure keratopathy varies depending on severity of the disease and underlying etiology. Primary treatments include lubrication with artificial tears and ointments, punctal plugs, short-term topical steroids, and topical immunosuppressives such as cyclosporine, bandage soft lenses, and amniotic membranes [5]. Long-term management may include moisture chamber use, lid taping, botulinum toxin injection, scleral lenses, and surgical options including permanent lateral tarsorrhaphy, upper eyelid weight placement, or lower lid reconstruction $[4,5]$. Often, combination treatments are prescribed to provide protection both day and night.

Exposure keratopathy can result from eyelid malposition after chemical burn, trauma, surgery, nerve damage including facial nerve palsy, ectropion, entropion, or proptosis due to thyroid orbitopathy [6]. Common clinical findings include impaired blink, lagophthalmos, decreased tear meniscus, decreased tear film break-up time, corneal filaments, punctate epithelial erosions, and epithelial defects which may cause a breakdown of epithelium and damage to corneal integrity [6-8]. Potentially sight threatening corneal edema, thinning, or ulceration may develop in severe cases $[5,6]$. When traditional therapies are inadequate, soft and scleral lenses can offer additional therapeutic effects [4, 9-12]. A study by Gervasio et al. [11] demonstrated benefits of PROSE, a custom designed and fabricated prosthetic device, for patients with postsurgical lagophthalmos. While PROSE treatment is limited to 12 US-based centers, commercially available scleral lenses are now widely available. A scleral lens is a largediameter custom-fit gas permeable lens that, unlike a traditional soft lens, provides a tear-filled vault bathing the ocular surface. In cases of inadequate eyelid function, therapeutic scleral lenses can provide a protective barrier from mechanical factors and corneal desiccation $[4,13]$. Although these patients may be managed primarily by an oculoplastic or corneal specialist, therapeutic scleral lenses should also be considered when surgical options are limited.

Therapeutic soft contact lenses and scleral lenses can decrease corneal discomfort, assist in corneal re-epithelialization, and protect the ocular surface from desiccation and mechanical factors [4, 13]. Both options can be considered for symptomatic patients when traditional therapies have failed. Therapeutic soft contact lenses can be beneficial in patients with severe dry eye or exposure keratopathy; however, in this patient, there were risks of contact lens drying and lens loss because of lack of eyelid integrity and support. Scleral lenses have many

\section{Karger'}


therapeutic benefits, as they can improve comfort, maintain constant lubrication to the ocular surface and simultaneously mask irregular astigmatism to improve visual acuity $[4,13]$. The post-lens tear reservoir of a scleral lens is filled with preservative-free saline solution prior to application which helps lubricate the cornea and supply it with proper nutrients [14].

Our patient had exposed cornea and conjunctiva due to complete loss of upper and lower eyelids and meibomian glands. His Bell's reflex was preserved, maintaining mechanical lubrication [15], which likely helped prevent corneal infection, ulceration, and perforation. Despite aggressive treatment with drops, ointment, and moisture chamber, he continued to report severe symptoms. Traditional lateral tarsorrhaphy was not an option for this patient due to loss of periocular tissue. Although additional surgery was discussed, it was deferred because of multiple concerns related to local vascular compromise and chronic tobacco use. In addition, our oculoplastics service felt that surgery alone would not resolve his ocular surface issues. Daytime use of a therapeutic scleral lens was prescribed for this patient with overnight moisture chamber use.

\section{Conclusion}

Patients with severe chemical burn and loss of eyelid function can have immediate relief of symptoms upon application of a scleral lens, improving quality of life. Therapeutic scleral lenses can also act as a bridge to provide immediate relief while additional surgery is scheduled. Using a therapeutic scleral lens, our patient has successfully maintained his occupation and lifestyle for 3 years and is currently planning additional reconstructive eyelid surgery. A team-based approach utilizing a therapeutic scleral lens should be considered in the management of severe chemical burn with exposure keratopathy.

\section{Statement of Ethics}

This project complies with the guidelines for human studies and was conducted ethically in accordance with the World Medical Association Declaration of Helsinski. Written informed consent was obtained from the patient for publication of this case report and accompanying images.

\section{Conflict of Interest Statement}

The authors do not have any conflicts of interest to declare.

\section{Funding Sources}

This project was supported by an unrestricted grant to the Department of Ophthalmology and Visual Sciences from Research to Prevent Blindness, NIH/NEI P30 EY001792, and NIH/ NEI K12 EY021475 (Scanzera). Article processing charges were covered by the University of Illinois at Chicago Research Open Access Aricle Publishing (ROAAP) Fund.

\section{Karger' ${ }^{\prime \prime}$}


Scanzera et al.: Therapeutic Scleral Lens after Severe Chemical Burn

\section{Author Contributions}

All the of the authors were involved in the clinical care of this patient. In addition, each author took part in drafting the work and critically revising it for important intellectual content.

\section{References}

1 McKee D, Thoma A, Bailey K, Fish J. A review of hydrofluoric acid burn management. Plast Surg. 2014;22(2): 95-8.

2 Singh P, Tyagi M, Kumar Y, Gupta KK, Sharma PD. Ocular chemical injuries and their management. Oman J Ophthalmol. 2013;6(2):83-6.

3 White ML, Chodosh J, Jang J, Dohlman C. Incidence of Stevens-Johnson syndrome and chemical burns to the eye. Cornea. 2015;34(12):1527-33.

4 Holland EJ, Mannis MJ, Lee WB. Ocular surface disease cornea, conjunctiva and tear film. London: Elsevier/ Saunders; 2013. Available only to UIC users: http://HZ9PJ6FE4T.search.serialssolutions.com/?V=1.0\& L=HZ9PJ6FE4T\&S=JCs\&C=TC0000967826\&T=marc\&tab=B00KS

5 Bizrah M, Yusuf A, Ahmad S. An update on chemical eye burns. Eye. 2019;33(9):1362-77.

6 Krachmer JH, Mannis MJ, Holland EJ. Cornea. 3rd ed. St. Louis, MO: Mosby/Elsevier; 2011.

7 Rahman I, Sadiq SA. Ophthalmic management of facial nerve palsy: a review. Surv Ophthalmol. 2007;52(2): 121-44.

8 Friedman NJ, Kaiser PK, Pineda R II. The Massachusetts eye and ear infirmary manual of ophthalmology. 5th ed. Print: Elsevier; 2019.

9 Scofield-Kaplan SM, Dunbar KE, Campbell AA, Kazim M. Utility of PROSE device in the management of complex oculoplastic pathology. Ophthalmic Plast Reconstr Surg. 2018 May-Jun;34(3):242-5.

10 Samimi DB, Chiu GB, Burnstine MA. PROSE scleral lens: a novel aid for staged eyelid reconstruction. Ophthal Plast Reconstr Surg. 2014;30(5):e119-21.

11 Gervasio KA, Godfrey KJ, Marlow ED, Lee MN, Lelli GJ Jr. Prosthetic replacement of the ocular surface ecosystem (PROSE) versus standard of care for postsurgical lagophthalmos and exposure keratopathy: trends in visual outcomes. Ophthalmic Plast Reconstr Surg. 2018.

12 Gire A, Kwok A, Marx DP. PROSE treatment for lagophthalmos and exposure keratopathy. Ophthal Plast Reconstr Surg. 2013;29(2):e38-40.

13 Barnett M, Johns KJ. Contemporary scleral lenses: theory and application. Sharjah: Bentham Science Publishers; 2018. Vol. 4.

14 Alipour F, Kheirkhah A, Jabarvand Behrouz M. Use of mini scleral contact lenses in moderate to severe dry eye. Cont Lens Anterior Eye. 2012;35(6):272-6.

15 Demirel S, Cumurcu T, Firat P, Aydogan MS, Doğanay S. Effective management of exposure keratopathy developed in intensive care units: the impact of an evidence based eye care education programme. Intensive Crit Care Nurs. 2014;30(1):38-44. 